\title{
Albert Scherr
}

\section{Offene Grenzen? \\ Migrationsregime und die Schwierigkeiten einer Kritik des Nationalismus}

Im Umgang mit unerwünschten Migrant/innen in den Staaten der EU wird in zugespitzter Weise der Widerspruch zwischen der Beanspruchung der Menschenrechte als Wertegrundlage und EU-europäisch bzw. nationalstaatlich gefassten politischen Interessen deutlich: Dass Migrant/innen als Subjekte ihrer Menschenrechte anzuerkennen sind, ist zwar prinzipiell - aber eben nur prinzipiell - unstrittig; dies verhindert aber Versuche der Abschließung der Außengrenzen mit der Folge zunehmend gefährlicher Fluchtwege ebenso wenig wie einen Umgang mit Asylsuchenden, Geduldeten und Illegalisierten, der - trotz der inzwischen in Deutschland verfassungsgerichtlich auferlegten Vorgaben ${ }^{1}$ - nach wie vor auf Abschreckung ausgerichtet ist. Verhindert werden dadurch auch nicht Abschiebungen in Kontexte, in denen Betroffene mit absoluter Armut, Diskriminierung und sozialer Ausgrenzung konfrontiert sind. ${ }^{2} \mathrm{Zu}$ verzeichnen sind in Folge einer Politik der Abschottung „Tausende und Abertausende von Toten, eine unbestimmte Anzahl von Vermissten, über die man nie mehr etwas erfahren wird“, und es ist durchaus plausibel, hierin eine „Nebenwirkung eines von Europa einseitig gegen Migranten erklärten Krieges“ (Paleologo 2008: 9) zu sehen.

Darauf reagieren unterschiedliche Formen der Kritik und der Solidaritätsarbeit mit Flüchtlingen, die von zivilgesellschaftlichen Organisationen, Wohlfahrtsverbänden und lokalen Initiativen getragen werden. In einer dem eigenen Anspruch nach radikalen Variante dieser Kritik wird die Legitimität staatlicher Migrationsregime generell in Frage gestellt und werden in der Folge ein unbegrenztes Recht auf Bewegungsfreiheit und Niederlassung sowie offene Grenzen und der

1 S. das Urteil des Bundesverfassungsgerichts vom 18.7.2012 (http://www.bverfg.de/entscheidungen/ls20120718_1bvl001010.html)

2 Und dies auch innerhalb Europas, wie im Fall von Roma, die aus Deutschland in den Kosovo, nach Serbien oder Rumänien abgeschoben (oder zur sog. freiwilligen Ausreise gedrängt) werden, dies nunmehr in Baden-Württemberg auch in Regie einer grün-roten Landesregierung. 
Verzicht auf Abschiebungen gefordert. ${ }^{3}$ Dies impliziert die Infragestellung eines konstitutiven Prinzips von Nationalstaatlichkeit, des Rechtes von Staaten, über den Zugang und Aufenthalt auf ihrem Territorium souverän entscheiden zu können, und damit eine Kritik, die über eine rassismustheoretische Kritik biologischrassistischer und ethnorassistischer Bestimmungen von Staatsangehörigkeit sowie instrumenteller politischer und ökonomischer Kalküle der Einwanderungspolitik notwendig hinaus geht. Denn bei einer grundsätzlichen Anerkennung des staatlichen Rechts, über Zugang zum und Aufenthalt auf dem Staatsgebiet zu entscheiden, können zwar die konkreten Modalitäten von Grenzsicherungsmaßnahmen, des Umgangs mit Flüchtlingen und der Abschiebepraxis kritisiert werden, nicht aber die prinzipielle Zulässigkeit der diese ermöglichenden Gesetze und der diese umsetzenden Institutionen und Praktiken. Folglich schließt der Verzicht auf eine generelle Kritik staatlicher Zuwanderungs- und Aufenthaltsregulierung auch eine implizite Legitimation von Grenzsicherungsmaßnahmen, innerstaatlichen Kontrollen und Abschiebungen ein. ${ }^{4}$

Insofern kann die Forderung nach offenen Grenzen für sich in Anspruch zunehmen, die einzig mögliche Position zum Ausdruck zu bringen, die sich einer solchen Legitimierung verweigert. Gleichzeitig handelt sich diese Position jedoch das Problem ein (s.u.), dass die Infragestellung der Zulässigkeit staatlicher Migrationsregime zugleich auch zu einer Infragestellung der Bestandsvoraussetzungen nationalstaatlich verfasster Demokratien und nationaler Wohlfahrtsstaaten führt, da diese als politische Vergemeinschaftung von Staatsbürger/innen sowie als Loyalitäts-Leistungs-Beziehungen zwischen Staaten und ihrer Bürger/innen konstruiert sind. ${ }^{5}$ In der Folge wird die Forderung nach offenen Grenzen in der sozialphilosophischen und sozialwissenschaftlichen Diskussion auch von Autor/ innen zurückgewiesen, die dem Spektrum der kritischen Theorien zuzurechnen sind, so u.a. von Etienne Balibar (2003), Seyla Behabib (2009) und Claus Offe (2011) (s.u.; vgl. Scherr 2012).

Vor diesem Hintergrund wird im Folgenden der Frage nachgegangen, ob und ggf. wie eine Kritik staatlicher Migrationsregime formuliert werden kann,

3 S. z.B. http://www.noborder.org; http://www.attac-netzwerk.de/ag-migration/texte/ offene-grenzen; http://www.migazin.de/2011/05/16/fur-ein-freies-europa-mit-offenengrenzen; http://www.transitmigration.org/db_transit/ausgabe.php?inhaltID=13;/

4 So fordert etwa die Partei 'Die Linke' „offene Grenzen für Menschen in Not“ (http://www.dielinke.de/index.php?id=251\&tx_ttnews[tt_news]=675\&tx_ttnews[backPid] $=35 \&$ no_ cache $=1$ ) und lässt damit die Fragen unbeantwortet, was als Not gelten soll sowie was mit denjenigen geschehen soll, die entsprechenden Kriterien nicht gerecht werden.

5 Darauf wird im Weiteren noch näher einzugehen sein; zur umfangreichen sozialwissenschaftlichen Diskussion über den Zusammenhang von Nationalstaatlichkeit, Wohlfahrtsstaatlichkeit und Migration s. Bommes/Halfmann 1998; Bommes/Geddes 2000. 
die sich nicht auf - die zweifellos legitime und erforderliche - moralische Empörung und menschenrechtliche Skandalisierung einzelner Fälle, Aspekte und Praktiken (etwa: der tödlichen Folgen des EU-Grenzregimes, der Drittstaatenregelung, der Lagerunterbringung, der Folgen von Illegalisierung, der Abschiebung unerwünschter Migrant/innen, der deutschen Residenzpflicht usw.) zurückzieht, sondern als Kritik der gesellschaftlichen Strukturen angelegt ist, die zu menschenrechtlich inakzeptablen Praktiken führen. ${ }^{6}$ Argumentiert wird hier diesbezüglich, dass es dazu nicht zureichend ist, eine rassismuskritische Position einzunehmen, sondern darüber hinausgehend erforderlich ist, eine Nationalismuskritik zu entwickeln, die auf die Forderung nach offenen Grenzen verzichtet, aber gleichwohl eine Delegitimation der staatlichen Entrechtung und Deklassierung unerwünschter Migrant/innen ermöglicht.

Dabei beanspruchen die folgenden Ausführungen nicht, eine abschließende Lösung der in Rede stehenden Problematik aufzuzeigen, sondern nur, zu einer Präzisierung der Frage beizutragen, mit welchen Schwierigkeiten Versuche konfrontiert sind, eine gesellschaftstheoretische Kritik staatlicher Migrationsregime zu konturieren.

\section{Globalisierung, Rassismuskritik und Staatsangehörigkeit}

Migrationsregime können als Artikulation eines strukturellen Widerspruchs der Weltgesellschaft verstanden werden (vgl. Stichweh 2000: 66ff.; Bommes 2011: 19ff.; Global Commission 2005):

Einerseits erzeugt der Prozess der Globalisierung unter Bedingungen weltweiter Ungleichheiten Migrationsanlässe, Migrationsmotive und Migrationsmöglichkeiten. Er etabliert einen Weltmarkt für Arbeitskräfte und umfasst die Aufforderung, auch jenseits des eigenen Herkunftslandes Qualifikationen zu erwerben und berufliche Möglichkeiten zu ergreifen; Kommunikationsmedien und ein globales Verkehrswesen lassen Migrationsmöglichkeiten sichtbar und erreichbar werden; bereits stattgefundene Migration erleichtert weitere Migration durch die

6 Die Menschenrechte werden hier ein als normativer Maßstab der Kritik beansprucht, der in den justiziell einklagbaren Menschenrechten keineswegs umfassend aufgehoben ist, sondern ein normativer Referenzrahmen für politische Auseinandersetzungen sind (s. dazu Ignatieff 2002); dabei wird davon ausgegangen, dass jede Kritik einen normativen Maßstab als Kriterium beanspruch muss, das es erlaubt, über die bloße Konstatierung von Tatsachen hinauszugehen und zu begründen, warum diese als inakzeptabel betrachtet werden (s. Ritsert 2009). Auf der grundlagentheoretischen Auseinandersetzung dazu, ob und ggf. wie eine nicht-normative Kritik möglich und erforderlich ist, kann hier nicht eingegangen werden. 
Entstehung von Netzwerken, usw. Mit dieser Erzeugung von Migrationsanlässen, -motiven und -möglichkeiten korrespondiert nicht zufällig ein - durchaus unwahrscheinlicher ${ }^{7}$ und kontroverser - Prozess, in dem Individuen als Träger von Menschenrechten anerkannt werden, die in Staatsbürgerrechten nicht umfassend aufgehoben sind (vgl. Koenig 2005): ${ }^{8}$ Unter Bedingungen einer fortschreitenden globalen Verflechtung von Ökonomie, Politik und medialer Kommunikation wächst der Bedarf an einem Referenzrahmen für internationale politische und rechtliche Kommunikation und damit steigt der politische Druck auf Staaten, sich auf die deklarierten Menschenrechte als einen solchen Referenzrahmen zu verpflichten. Zudem ist unter Bedingungen fortschreitender Globalisierung die Vorstellung einer „Universalgesellschaft, die die ganze Menschheit umfasst und in der alle durch das Mittel der Kommunikation Beziehungen zueinander aufnehmen können“(Mead 1934/1968: 330) nicht mehr nur als philosophisches Konzept relevant, sondern zunehmend in Alltagserfahrungen verankert; damit wird es auch wahrscheinlicher, dass eine Anerkennung Anderer als Mitglieder dieser Universalgesellschaft, die einander moralisch verpflichtet sind (ebd.), möglich wird. Damit werden die gesellschaftsstrukturellen Grundlagen einer nationalstaatlichen Begrenzung von politischer, rechtlicher und moralischer Zuständigkeit brüchig (siehe dazu auch Fraser 2010).

Andererseits gilt jedoch das Recht von Staaten, über den Zugang zu und Aufenthalt auf ihrem Territorium sowie den Erwerb von Staatsbürgerschaft souverän zu entscheiden, also das Recht, Migration zu begrenzen und zu regulieren, im dominanten politischen Diskurs und auch in weiten Teilen des sozialwissenschaftliches Diskurses als nicht aussichtsreich kritisierbare Faktizität. Denn Globalisierung führt nicht zur Auflösung von „Nationalgesellschaften“ (Schimank 2005: 399) in Richtung einer Weltgesellschaft, sondern 'nur' dazu, dass deutlicher erkennbar wird, dass es sich bei Gesellschaften mit einem nationalstaatlich verfassten politischen System nicht um selbstgenügsame Einheiten

7 Unwahrscheinlich ist dieser Prozess deshalb, weil er die Bindung moralischer und rechtlicher Verpflichtungen von der Zugehörigkeit zu einer partikularen Gruppe entkoppelt; siehe zu dieser Problematik in Hinblick auf die wohlfahrtsstaatliche Inklusion von Zuwanderern die bei Mau und Burkhardt (2010) dargestellten Forschungsergebnisse.

8 Dass Individuen als Menschen gelten und dass ihnen schon daraus Rechte erwachsenen, welche die Möglichkeiten staatlicher Herrschaftsausübung begrenzen, war und ist keineswegs selbstverständlich, sondern Ergebnis eines historischen Lernprozesses, der aus der Auseinandersetzung mit Unrechtserfahrungen resultiert (vgl. Bielefeld 1998). Die Etablierung eines Menschenrechtsdiskurses verhindert zwar faktische Menschenrechtsverletzungen und die politische Instrumentalisierung der Menschenrechtsidee nicht; jede Kritik der Beanspruchung der Menschenrechte als Maßstab der Kritik müsste aber aufzeigen können, welchen alternativen, der Menschenrechtsidee überlegenen Maßstab sie stattdessen beansprucht. 
handelt, sondern um ein „Resultat relativer Abschottung“ (ebd.: 399). Genauer: Um das Resultat eines hoch selektiven Arrangements von Öffnungen und SchlieBungen für Kapital, Geld, Waren, Dienstleistungen, das historisch und aktuell Versuche der Mobilisierung und Demobilisierung von Menschen einschließt (vgl. Bauman 2005: 90ff) . Unter Bedingungen globaler Ungleichheiten sind Nationalstaaten insbesondere dadurch auch als ,institutionalisierte Gleichheits/Ungleichheitsschwellen“ (Stichweh 2000: 69: 52) bedeutsam, dass der Zugang zu rechtlich regulierten Arbeitsmärkten sowie zu wohlfahrtsstaatlichen Leistungen, aber auch zu rechtsstaatlichen Garantien und demokratischer politischer Interessenvertretung von der Staatsangehörigkeit und dem Aufenthaltsstatus abhängig ist und folglich durch Migrationsregime reguliert wird.

Der strukturelle Widerspruch zwischen der Erzeugung von Zwängen, Anreizen und Möglichkeiten für Migration sowie der Anerkennung von Menschen als Subjekte, für deren Schicksal nicht exklusiv ihr Herkunftsstaat zuständig ist einerseits, andererseits einer staatlich-politischen Regulierung von Migration, die nationalstaatlich (bzw. EU-europäisch) gefasste Interessen ins Zentrum stellt, ist im politischen und medialen Diskurs, der Migrationsforschung und rassismuskritischen Analysen wiederkehrend thematisiert worden. Deutlich wurde dabei, dass Migrationsregime auf einer doppelten Diskriminierung beruhen: Auf der politisch-rechtlichen Regulierung von Zuwanderung einerseits, der Zuweisungen benachteiligender Positionen in den jeweiligen Einwanderungsgesellschaften andererseits. Insofern ist es naheliegend, die Kontinuität zwischen gegenwärtiger staatlicher Diskriminierung von Migrant/innen und historischen Formen von Rassismus zu betonen (vgl. etwa Balibar 1990: 49ff.). Gleichwohl kann nicht davon abgesehen werden, dass an das Kriterium der Staatsangehörigkeit geknüpfte Formen der Diskriminierung keinesfalls notwendig mit biologisch-rassistischen und/oder ethnischen Konstruktionen legitimer Zugehörigkeit und ungleicher Rechte einhergehen müssen. Denn Staatsangehörigkeit kann als folgenreiche rechtliche Kategorie in einer Weise gefasst werden, welche die Argumente der Rassismuskritik aufnimmt.

Dies wird in Deutschland u.a. in der Überwindung eines exklusiven jus sanguinis deutlich, die dazu geführt hat, dass ein dauerhafter Aufenthaltsstatus und die Staatsbürgerschaft, und damit Zugangsrechte zum Arbeitsmarkt und politische Partizipationsrechte, unter bestimmten Bedingungen auch erworben werden können, ohne dass biologisch-rassistische und kulturrassistische Kriterien diese Bedingungen definieren. Auf der Ebene der EU geht eine Politik der verschärften Kontrolle von Zuwanderung mit einer Antidiskriminierungsprogrammatik einher, die jede Benachteiligung aufgrund „der Rasse oder der ethnischen Herkunft (RICHTLINIE 2000/43/EG DES RATES) untersagt. Explizit ausgenommen vom Diskriminierungsverbot sind jedoch die „Ungleichbehandlungen aufgrund 
der Staatsangehörigkeit“ und damit „Vorschriften über die Einreise und den Aufenthalt von Drittstaatsangehörigen und ihren Zugang zu Beschäftigung und Beruf“ (ebd.: Art. 13). Anerkannte Grenzen des Rechts, Nicht-Staatsangehörige bzw. Nicht-EU-Bürger/innen auszugrenzen und zu benachteiligen, sind allein die restriktiven Bestimmungen der Asylgesetzgebung sowie das Non-RefoulementPrinzip der Genfer Flüchtlingskonvention.?

Damit wird in der Anti-Diskriminierungsgesetzgebung, wie auch in den korrespondierenden Diversity- und Anti-Rassismus-Programmatiken, exemplarisch die Idee eines modernen Europa sichtbar, das sich von tradierten ideologischen Formen der Diskriminierung distanziert und dadurch in der Lage ist, sich als den Menschenrechten verpflichtete Wertegemeinschaft zu beschreiben. Ich tendiere diesbezüglich zu der Einschätzung ${ }^{10}$, dass die Interessen der politischen Eliten der EU und der global operierenden ökonomischen Eliten darauf ausgerichtet sind, einen Modernisierungsprozess voranzutreiben, für den sich rassistische und ethnische Unterscheidungen (aber auch geschlechtsbezogene Diskriminierung) und darin begründete Konflikte als Hindernis für die ökonomische Nutzbarmachung von Humankapital sowie die Expansion der Absatzmärkte darstellen und der deshalb auf eine Purifizierung des meritokratischen Versprechens zielt, dass jede/r - unabhängig von Herkunft, Geschlecht, sexueller Orientierung, Ethnizität usw. - formell gleiche Chancen in der ökonomischen Leistungskonkurrenz haben soll. Antidiskriminierungspolitik ist so betrachtet ein zentraler Ausdruck dieses gegenwärtig hegemonialen Projekts. Damit wird ein Bündnis mit emanzipatorischen sozialen Bewegungen und kritischer Sozialwissenschaft möglich. Dies jedoch nur in einem Rahmen, in dem zwei gravierende Formen von struktureller Benachteiligung systematisch ausgeklammert bleiben: Die ökonomischen Ungleichheitsverhältnisse, die aus den ökonomischen Strukturen innerhalb der EU und ihrer Nationalstaaten resultieren sowie die staatlichpolitisch, nicht zuletzt durch Migrationsregime, insbesondere eine restriktive Flüchtlingspolitik und eine selektive Politik der Arbeitsmigration abgesicherten Ungleichheitsverhältnisse zwischen den Regionen der Weltgesellschaft. Denn nicht die Struktur der internationalen und innernationalen Ungleichheiten, sondern nur diskriminierende Praktiken bei der Zuweisung von Positionen in

9 Dies hat zur Folge, dass sich die Auseinandersetzung über das Aufenthaltsrechts von Flüchtlingen immer wieder von der Ebene politischer Entscheidungen auf die komplexer juristischer Abwägungen verlagert, in denen dann z.B. umfangreiche Gutachten zu der Frage entscheidungsrelevant sind, welche Krankheiten in welchen Regionen der Welt angemessen behandelt werden können.

10 Diskursanalysen, die diese Einschätzung fundieren könnten, liegen meiner Kenntnis nach bislang nicht vor. 
diesen Strukturen selbst sind im hegemonialen Diskurs als kritikbedürftig anerkannt (vgl. Scherr 2010).

Vor diesem Hintergrund soll im Weiteren danach gefragt werden, wie eine Kritik EU-europäischer und nationalstaatlicher Migrationsregime formuliert werden kann, die sich dieser systematischen Ausklammerung verweigert und den universalistischen Anspruch des menschenrechtlichen Diskriminierungsverbots ernst nimmt: Wie kann eine Kritik von Verhältnissen formuliert werden, in denen - jedenfalls de jure - niemand innerhalb der EU rassistisch, aufgrund von Ethnizität, „Rasse“, Religion usw. - diskriminiert werden darf, in denen es aber als zulässig gilt, Staatsbürgerschaft als Diskriminierungskriterium zu verwenden? Kann die Verwendung von Staatsbürgerschaft in gleicher Weise kritisiert werden, wie die Verwendung rassistischer und ethnisierender Unterscheidungen? ${ }^{11}$

\section{Nationalstaatliche Vergesellschaftung und Nationalismus}

Um diese Fragen angemessen bearbeiten zu können, ist es erforderlich, zwischen einem ideologisch aufgeladenen Nationalismus und einem eher sachlich-nüchternen, funktionalen Nationalismus zu unterscheiden. Historisch war zwar eine Konstruktion von Nationalstaaten als „,imaginäre Gemeinschaften“ für die Staatenbildung in Europa unverzichtbar - und damit einhergehend ein ideologisch aufgeladener Nationalismus, der Nationen als intern homogene und deshalb nach außen abgrenzbare Kollektive konzipiert sowie entsprechende Praktiken der internen Homogenisierung, einschließlich der Vertreibung und Vernichtung von Minderheiten, realisiert hat. Demgegenüber lassen sich gegenwärtig Entwicklungen beschreiben, in deren Folge ein solcher Nationalismus in EU-Europa als Grundlage hegemonialer Politik in Frage gestellt ist (siehe Bauman 2003: 216ff.; Castells 1997: 5ff.) und in der Folge als Merkmal eines als unzeitgemäß geltenden Rechtspopulismus gilt: ${ }^{12}$ Die Rolle von Nationalstaaten verändert sich. Sie sind nicht mehr souveräne Subjekte, sondern nur noch strategische Akteure im Kontext einer globalen Ökonomie; im Zuge der Verwandlung der europäischen Nationalstaaten in Einwanderungsgesellschaften, der Verlagerung

11 Zur Verdeutlichung: Eine Abschiebung von Roma aus Deutschland wäre rechtswidrig, wenn sie den Verweis auf die ethnische Kategorie zur Begründung verwenden würde; als legal gilt dagegen die Abschiebung kosovarischer und serbischer Staatsbürger/innen - unabhängig davon, ob es sich um Roma handelt oder nicht, da die Bundesländer die Anerkennung einer gravierenden kollektiven Diskriminierung verweigern.

12 Zweifellos sind sind auch Prozesse der Tradierung eines ethno-nationalistischen Alltagsbewusstseins sowie Gegentendenzen zur Erosion des ideologisch aufgeladenen Nationalismus zu beobachten. 
nationalstaatlicher Zuständigkeiten auf die Ebene der EU, von kultureller Globalisierung und der Etablierung eines Menschenrechtsdiskurses wird die Grundlage ethnischer und rassistischer Konstruktionen nationaler Einheit brüchig. Und eine starke Identifikation mit der eigenen Nation in Verbindung mit einem Überlegenheitsbewusstsein ist nach vorliegenden Umfrageergebnissen zwar keineswegs überwunden, aber, jedenfalls in Deutschland, aktuell nicht mehrheitsfähig (vgl. Decker/Brähler 2006). ${ }^{13}$

Die Beobachtung von Tendenzen, die dazu führen, dass der „Begriff einer nationalen Identität, mit der ein Einzelner sich identifizieren kann, die Plausibilität“ entzogen wird und die Annahme, dass ,wir uns heute in einer Auslaufphase dieser Idee befinden" (Luhmann 1997: 1055), treffen jedoch nicht in gleicher Weise auf einen solchen Nationalismus zu, der auf tradierte ideologische Aufladungen verzichtet und nicht mehr beansprucht, als die politische und rechtliche Legitimität der historisch gewordenen Grenzen, der historisch gewordenen Zusammensetzung der Bevölkerung sowie der gegebenen Unterschiede und Ungleich heitsverhältnisse zwischen den Nationalstaaten. Denn dieser Nationalismus - der auch immer wieder erneut Bestimmungen nationaler Identität erforderlich werden lässt und damit Versuche der ideologischen Aufladung veranlasst - hat nach wie vor eine gesellschaftsstrukturelle Grundlage: Staatliche Vergesellschaftung ist auch unter Bedingungen fortgeschrittener Globalisierung immer noch hoch folgenreich für die individuellen Lebensbedingungen und erzeugt damit objektive Interessenlagen der als Staatsbürger/innen vergesellschafteten Individuen. ${ }^{14}$ Denn Staaten konstituieren „Nationalgesellschaften“ (Schimank 2005) als politische Einheiten in der Weltgesellschaft, die den (legalen) Zugang zu Arbeitsmärkten, Bildungssystemen, Gesundheitssystemen, Sozialleistungen, politischer Partizipation und Rechtssystemen regulieren. Als Staatsbürger/in eines EU-Staates ist man folglich faktisch gegenüber den Bürger/innen der meisten außereuropäischen Staaten in verschiedener Hinsicht privilegiert und damit objektiv auch dann an der eigenen Staatszugehörigkeit interessiert, wenn man nationalistische Identifikationszumutungen ablehnt. ${ }^{15}$ Zudem sind Staatsgrenzen auch als Grenzen der "Informations- und Betroffenheitshorizonte" (Schimank 2005: 401) folgenreich: Schulische Bildung, mediale und politische Kommunikation adressieren ihr Publikum als Staatsbürger/innen und sie reduzieren Komplexität nicht zuletzt dadurch, dass sie Ereignissen im jeweiligen nationalstaatlichen Kontext primäre

13 Dies wird nicht zuletzt in der abnehmenden Bereitschaft deutlich, sich für nationale Armeen rekrutieren zu lassen und ggf. für's „Vaterland“ zu sterben.

14 Dies gilt ganz analog zu Klassenlagen und darin begründeten objektiven Interessen.

15 Selbst politische Aktivist/innen achten deshalb gewöhnlich sehr darauf, dass ihnen bei Reisen in Länder der Peripherien der Reisepass nicht abhanden kommt. 
Bedeutung zusprechen. In der Folge der wiederkehrenden Anrufung von Individuen als nationale Subjekte erscheint die Unterteilung der Weltgesellschaft in Staaten und Staatsbevölkerung trotz fortgeschrittener Globalisierung als die fraglos-selbstverständliche, quasi-natürliche und alternativlose Ordnung der Dinge.

Dem korrespondiert ein „gewöhnlicher Nationalismus“ (Pogge 2011: 152ff.), der nicht mehr voraussetzt als die stillschweigende Akzeptanz gegebener staatlicher Strukturen und die Legitimität einer an staatlich gefassten Interessen orientierten Politik. Thomas Pogge (2011) zufolge ist für diesen gewöhnlichen Nationalismus nicht mehr als die Annahme erforderlich, dass sich „Bürger und Regierungen (...) stärker um Überleben und Wohlergehen ihres eigenen Staates, ihrer Mitbürger und ihrer Kultur kümmern“ dürfen und sollen, „als um Überleben und Wohlergehen fremder Staaten, Kulturen und Personen" (ebd.: 151). Der "gehobene Nationalismus“ (ebd.) ist zudem dadurch gekennzeichnet, dass er zwar mit der Forderung nach einer gerechten Gestaltung der Lebensverhältnisse einhergeht, daraus aber vorrangig eine diesbezügliche Verpflichtung in Bezug auf den eigenen Staat und seine Bürger/innen ableitet. Es handelt sich bei diesen Varianten des Nationalismus Pogge zufolge um einen weitverbreiteten „konservativen Nationalismus" (150) der Bürger/innen in den wohlhabenden Weltregionen: „Diese Personen sind mit dem Status quo (der Weltgesellschaft, A. S.) recht zufrieden und betrachten es als legitim oder gar anerkennenswert, dass sie und ihre politischen Vertreter sich überwiegend darum kümmern, ihren eigenen kollektiven Vorteil zu wahren und zu vergrößern. Die meisten Bürger der wohlhabenden Staaten sind ... Nationalisten, und zwar extreme Nationalisten, in diesem Sinne." (150)

Ein solcher extremer Nationalismus - und das ist hier entscheidend - benötigt jedoch keine explizite ethno-nationalistische oder rassistische Ideologie und auch keine Konstrukte der nationalen Überlegenheit. Er kann durchaus mit einer explizit multikulturellen oder einer republikanischen Konzeption von Nationalstaatlichkeit ${ }^{16}$ und einer Kritik innerstaatlicher Formen der Diskriminierung von Migrant/innen und Minderheiten einhergehen. Zu seiner Rechtfertigung ist nicht mehr als die Annahme erforderlich, dass die vermeintlichen oder tatsächlichen Interessen der nationalstaatlich vergesellschafteten Bürger/ innen als solche legitim sind sowie dass nationalstaatliche Politik berechtigt ist, sich primär an den Interessen „ihrer“ Bürger/innen zu orientieren. Deshalb reicht eine Kritik rassistischer und kulturrassistischer Ideologien und Diskurse an den Kern dieses Nationalismus nicht heran. Eine Kritik des konservativen

16 So im Fall von Kanada bzw. Frankreich. 
Nationalismus kann vielmehr nur dann formuliert werden, wenn die moralische und politische Legitimität der historisch gewordenen Ungleich heitsverhältnisse zwischen den Nationalstaaten und der Praktiken in Frage gestellt wird, durch die diese abgesichert werden.

\section{Sind staatliche Grenzziehungen kritisierbar?}

Eine darauf ausgerichtete Kritik ist meines Erachtens unverzichtbar, etabliert aber erhebliche Schwierigkeiten. Unverzichtbar ist diese Kritik, weil auch der konservative und gehobene Nationalismus faktisch die Legitimationsgrundlage einer deutschen und EU-europäischen Politik ist, die soziale Gerechtigkeit primär in einem nationalstaatlichen bzw. EU-europäischen Rahmen konzipiert und in der Folge im Umgang mit Flüchtlingen jede Berufung auf die Menschenrechte als Artikulation einer universalistischen Moral konterkariert (vgl. Weinzierl/ Lisson 2007). Und der Widerspruch einer Weltgesellschaft, die Migrationsmotive und Migrationsmöglichkeiten erzeugt, die als universell proklamierten Menschenrechte als normativen Konsens beansprucht, aber zugleich an ökonomischen und politischen Interessen orientierte staatliche Begrenzungen der Migrationsmöglichkeiten vornimmt, lässt sich auch nicht zureichend durch das Asylrecht und die Bestimmungen der Genfer Flüchtlingskonvention aufösen. Denn mit welchen legitimen Gründen ist Menschen das Recht zu bestreiten, aus Gesellschaften auszuwandern, in denen sie keinen zureichenden Zugang zu ausreichender materieller Versorgung, Bildung und Gesundheit finden? Oder: Wie kann es legitimiert werden, dass innerhalb der EU Migration prinzipiell als zulässig gilt, nicht aber die Zuwanderung aus Drittstaaten in die EU? Auch gerechtigkeitstheoretisch ist anzuerkennen, dass die weitreichenden Folgen des Schicksals der Geburt in einem Staat, die Folgen der „birthright lottery“ (Sachar 2009) nicht rechtfertigbar sind. Denn mit guten Gründen lässt sich argumentieren, dass die Privilegien, die aus dem nationalen Geburtsort resultieren, das moderne Äquivalent der mittelalterlichen Feudalordnung sind, die als quasiständische Festlegungen von Lebenschancen den Kernprinzipien moderner Vergesellschaftung widersprechen (Bauböck 1997).

Thomas Pogge (2011: 177ff.) hat - in Auseinandersetzung mit Rawls Theorie der Gerechtigkeit - zudem überzeugend argumentiert, dass die Begrenzung staatlich-politischer Verpflichtungen auf die Bürger/innen des eigenen Staates auch deshalb nicht rechtfertigbar ist, weil die Ursachen des in weiten Teilen der Welt herrschenden Elends nicht unabhängig sind von der Politik, insbesondere der Wirtschaftspolitik der entwickelten Nationen. Wenn z.B. die EU und die USA durch Subventionen ihrer Agrarindustrie dazu beitragen, dass landwirt- 
schaftliche Produktion andernorts unrentabel wird, können sie moralische und politische Verantwortung für die Situation derjenigen, die dadurch in absolute Armut geraten, nicht zurückweisen.

Insofern liegt es unter Berufung auf die universalistischen Prinzipien der Menschenrechte und gerechtigkeitstheoretisch nahe, offene Grenzen zu fordern und die Legitimität staatlicher Migrationsregime zu bestreiten: Jede/r soll demnach die Chance haben, durch Migration seine Lebensbedingungen zu verbessern, und dies nicht nur innerhalb eines Staates oder innerhalb der EU, sondern weltweit. Diese Forderung ist aber nicht nur im politischen Diskurs randständig. Sofern sie überhaupt ernsthaft diskutiert wird (siehe Carens 1987; Bauböck 1997; Offe 2011), wird sie auch von Vertreter/innen kritischer Sozialwissenschaft überwiegend zurückgewiesen. Diskussionsbedürftig sind dabei - neben z. T. recht durchsichtigen Rechtfertigungsversuchen - m. E. vor allem zwei Argumentationslinien:

Erstens wird argumentiert, so etwa bei Michael Walzer (1992), dass demokratisch verfasste politische Gemeinschaften historisch und systematisch an die Form des Nationalstaates gebunden sind und deshalb ein Recht auf Regulierung von Zugehörigkeit aus dem Selbsterhaltungsinteresse von Demokratien abgeleitet werden kann. In dieser Argumentationslinie, die auch Seyla Behabib aufgreift (2009: 118ff. und 137ff.), hat zwar jedes Individuum ein Recht auf Staatsangehörigkeit und auch das Recht auf Zugehörigkeit zu einem demokratisch verfassten Staat. Dieses Recht wird jedoch als einschränkbar betrachtet, sofern Einschränkungen nicht willkürlich, sondern begründet und in Verfahren überprüfbar sind. Behabib (2009: 140) argumentiert wie folgt: Sie reklamiert ein „Menschenrecht auf Zugehörigkeit“, das „über die konkrete Einbürgerungsgesetzgebung dieses oder jenes Landes hinausgeht“. Gleichzeitig erkennt sie das Recht jedes Landes an, unterschiedliche Bedingungen für Einbürgerungen festzulegen. „Solche Variationen sind Sache der jeweiligen Nation." Damit wird die Verknüpfung von Territorialstaatlichkeit und Demokratie und damit das Recht demokratischer Staaten auf Zuwanderungsbegrenzung und Zugehörigkeitsregulierung demokratietheoretisch legitimiert: „Da die in Demokratien geltenden Gesetze für ihre Urheber bindend sein sollen, ist ihre Legitimation an den demos gebunden, der sich als souveränes Volk auf einem bestimmten Territorium konstituiert. Die Verantwortung für die Gesetze trägt das Volk, und dessen demokratische Repräsentation setzt eine Abschließung nach außen voraus. (..) Imperien haben Fronten. Demokratien haben Grenzen." (ebd.:211)

Eine zweite Argumentationslinie (Balibar 2003: 201ff.; Bommes 1992; Bommes/Geddes 2000; Offe 2011) verweist auf den konstitutiven Zusammenhang von Nationalstaatlichkeit und Wohlfahrtsstaatlichkeit. Diese Argumentationslinie kann pointiert wie folgt zusammengefasst werden: Migration ist nicht zuletzt durch die internationalen Ungleichheitsverhältnisse veranlasst, die durch nati- 
onale Wohlfahrtsstaaten verfestigt werden. Wohlfahrtsstaatlichkeit beruht auf nationalstaatlich konturierten Leistungs- und Loyalitätsbeziehungen zwischen Staaten und ihren Bürger/innen sowie auf Begrenzungen des Zugangs zu Arbeitsmärkten, Bildungssystemen, Gesundheitssystemen und Sozialleistungen. Wer für offene Grenzen plädiert, muss folglich auch für einen Abbau bzw. eine erhebliche Einschränkung wohlfahrtsstaatlicher Leistungen eintreten. Zugespitzt formuliert: Nur ein komplett deregulierter Kapitalismus benötigt keine Zuwanderungsbegrenzungen. Oder mit einer Formulierung von Etienne Balibar (2003: 286): Eine Welt ohne Grenzen würde „zur Ausweitung eines Raubtierkapitalismus“ führen, ,in dem die Menschen endgültig wie Waren ... von den Produktionsstandorten angezogen und abgestoßen würden." Dieses Argument ist in dem Maß plausibel, wie eine Begrenzung der ökonomischen Macht global operierender Konzerne nur durch staatliche Politik bzw. im Kontext nationalstaatlich gerahmter politischer Auseinandersetzungen denkbar ist und eine nicht an die Form der nationalstaatlich gebundenen Gestaltung von sozialer Sicherung, allgemeiner Bildung usw. als unrealistisch erscheint. Insofern handelt sich die Forderung nach offenen Grenzen einen Konflikt zu den legitimen Eigeninteressen derjenigen ein, die als Bürger/innen der westlichen Wohlfahrtsstaaten ein Interesse an staatlich-politischer Regulation ihrer Lebensbedingungen haben.

Vor dem Hintergrund dieser und weiterer Argumente, die hier nicht diskutiert werden können, ${ }^{17}$ stellt sich die Frage, ob eine Kritik des funktionalen konservativen Nationalismus zwar menschenrechtlich legitim, aber insofern hinfällig ist, weil sie in eine „Negation ohne Alternativkonzept“ (Luhmann 1997: 1119) mündet. Eine solche Kritik ist zwar zweifellos legitim, bleibt aber politisch folgenlos, wenn nicht aufgezeigt werden kann, was eine andere und anstrebenswerte Form der Vergesellschaftung wäre, die an die Stelle des kritisierten Zustands treten kann. Ergeht es der Kritik des Nationalismus also wie (zumindest bislang) der Kritik des Kapitalismus: Sie kann gute Gründe für sich beanspruchen, aber keine realistische sowie durchsetzbare Alternative zum kritisierten Zustand aufzeigen? Bleibt also nichts übrig, als das Paradox auszuhalten, dass es gute Gründe gibt, den herrschenden Nationalismus unter menschenrechtlichen und gerechtigkeitstheoretischen Gesichtspunkten zu kritisieren, und zugleich anzuerkennen, dass Menschenrechte und soziale Gerechtigkeit faktisch nur im nationalstaatlichen Rahmen gewährleistet werden können? Müssen also auch kritische Sozialwis-

17 Claus Offe (2011) argumentiert u.a., dass offene Grenzen auch deshalb nicht dazu geeignet sind, die strukturellen Probleme der Weltgesellschaft zu lösen, weil sie mit hoher Wahrscheinlichkeit (Abwanderung der Jungen und Qualifizierten, ökonomische Abwärtsspirale) auch zu einer Verschärfung der Probleme in den Auswanderungsländern führen würden. 
senschaftler/innen und Aktivist/innen in sozialen Bewegungen die prinzipielle Legitimität des europäischen Migrationsregimes anerkennen und sich auf eine punktuelle Kritik rechtlicher Festlegungen und ihrer Realisierung begrenzen? Die Konsequenzen, die aus einer bejahenden Antwort auf diese Fragen folgen, sind $\mathrm{m}$. E. analytisch kaum zu vermeiden, aber zugleich normativ inakzeptabel. Deshalb ist es erforderlich, über Perspektiven der Kritik nachzudenken, die davon ausgeht, dass es vor dem Hintergrund der historisch gewordenen Verhältnisse keine absehbare Auflösung der Widersprüche gibt, die sich im Umgang mit unerwünschten Migrant/innen in zugespitzter Weise artikulieren.

\section{Perspektiven der Kritik}

Kritik steht darauf bezogen meines Erachtens erstens vor der Aufgabe, die Widersprüche zwischen der Berufung der jedem Einzelnen zukommenden Menschenrechte ${ }^{18}$ sowie die Idee sozialer Gerechtigkeit und dem Umgang mit Flüchtlingen unter Bedingungen fortgeschrittener Globalisierung als solche zu thematisieren und zu einer offenen Auseinandersetzung mit diesen Widersprüchen herauszufordern. Dazu ist es erforderlich, einen öffentlichen Diskurs in Gang zu setzen bzw. fortzuführen, in dem deutlich zu einer Debatte über das Verhältnis der vermeintlichen und tatsächlichen Interessen nationalstaatlich vergesellschafteter Bürger/innen zu menschenrechtlichen Grundsätzen und den darin begründeten Ansprüchen von Flüchtlingen aufgefordert wird. In dem Maße, wie eine offene, die Interessengegensätze nicht verschleiernde Auseinandersetzung über das Verhältnis von nationalstaatlich gefassten Interessen und den Prinzipien einer universalistischen Moral in Gang gesetzt werden kann, besteht die Chance, den gewöhnlichen Nationalismus zu verunsichern.

Dazu ist es zweitens unverzichtbar, Varianten eines explanatorischen Nationalismus zu kritisieren, der die Ursachen unzumutbarer Lebensbedingungen exklusiv in den jeweiligen nationalgesellschaftlichen Verhältnissen der Auswanderungsgesellschaft sieht und damit Verantwortlichkeit für ihre Folgen zurückweist. Aufzuzeigen sind demgegenüber die Verstrickungen europäischer Politik und Ökonomie in die Hervorbringung von Fluchtursachen. Für die sozialwissenschaftliche Forschung und Theoriebildung schließt dies eine Problema-

18 Der gegen eine erste Fassung dieses Textes erhobene Einwand, dass hier ein politisch naives Verständnis der Menschenrechte in Anspruch genommen würde, ist m. E. nicht tragfähig; denn erst die Beanspruchung der Menschenrechte als normativer Horizont politischer Entscheidung ermöglicht es, Verpflichtungen staatlicher Politik gegenüber Nicht-Staatsbürgern einzufordern (siehe dazu u.a. Fraser 2010). 
tisierung der etablierten wissenschaftlichen Arbeitsteilung ein, insbesondere die Abkoppelung der Diskurse über Migrationsregime und Migrationspolitik von der wissenschaftlichen Beschäftigung mit Fragen der globalen Ungleichheit, der Außenpolitik, Handelspolitik und der Entwicklungspolitik. Denn die Abkoppelung der Migrationsforschung von weiterreichenden gesellschaftstheoretischen Fragestellungen bedingt eine Spezialisierung, in deren Folge die übergreifenden Zusammenhänge aus dem Blick treten können.

Drittens ist es erforderlich, die argumentative Falle zu vermeiden, die entsteht, wenn allein der Umgang mit Migrant/innen und Flüchtlingen im Zentrum von Auseinandersetzungen über Erfordernisse einer menschenrechtlich legitimen Politik unter Bedingungen globaler Ungleich heiten steht. Denn es ist plausibel zu argumentieren, dass die Aufnahme von Flüchtlingen nur begrenzt dazu geeignet ist, die Folgeprobleme globaler Ungleichheit zu lösen. Folglich sind die Kritik von Außen-, Entwicklungs-. und Handelspolitik und die Kritik von Migrationsregimen als einander notwendig ergänzende zu begreifen.

Viertens ist eine Form der Kritik weiterzuentwickeln, die für sich nicht beansprucht, über generelle und abschließende Lösungen zu verfügen, aber in Bezug auf je konkrete Fälle und Aspekte die konsequente Beachtung menschenrechtlicher Gesichtspunkte einfordert; eine Kritik, welche die Gewalt der Abstraktion in den Blick rückt, die darin besteht, dass das Schicksal konkreter Einzelner hinter die Betrachtung der generellen Problematik des Umgangs mit Migration unter Bedingungen globaler Ungleich heiten zurücktritt. Eine solche Kritik, die den menschenrechtlichen Grundsatz der zu achtenden Würde jedes Einzelnen in seiner Radikalität ernst nimmt, kann idealiter dazu beitragen, dass die wiederkehrende und organisierte Verdrängung des Schicksals von Flüchtlingen immer wieder aufgebrochen wird.

\section{Literatur}

Balibar, Etienne (1990): Rassismus und Nationalismus. In: Etienne Balibar/Immanuel Wallerstein: Rasse - Klassen - Nation. Hamburg, S. 49-84.

- (2003): Sind wir Bürger Europas? Hamburg.

Bauböck, Rainer (1997): Gehen, Bleiben, Kommen. Notwendige Öffnung und legitime Schließung liberaler Demokratien. in: Archives Européennes de Sociologie, No.1, 71-103.

Bauman, Zygmunt (2003): Flüchtige Moderne. Frankfurt/M.

- (2005): Verworfenes Leben. Frankfurt/M.

Behabib, Seyla (2009): Die Rechte der Anderen. Bonn.

Bielefeldt, Heiner (1998): Philosophie der Menschenrechte. Darmstadt.

Bommes, Michael (1992): Migration in nationalen Wohlfahrtsstaaten. Osnabrück.

- (2011): Nationale Paradigmen der Migrationsforschung. In: Ders.: Migration und Migrationsforschung in der modernen Gesellschaft. Osnabrück (IMIS-Beiträge 38/2011), $15-52$.

Bommes, Michael/Halfmann, Jost (Hrsg.) (1998): Migration in nationalen Wohlfahrtsstaaten. Osnabrück. 
Bommes, Michael/Geddes, Andrew (Eds.) (2000): Immigration and Welfare: Challenging the Borders of the Welfare State. London.

Carens, Joseph H. (1987): Aliens and Citizens: The case of open borders. In: The Review of Politics, Vol 49, No. 2, 251-273.

Castells, Manuel (1997): The Power of Identity. Oxford.

Decker, Oliver/Brähler, Elmar (2006): Vom Rand zur Mitte. Berlin.

Fraser, Nancy (2010): Who counts as a Subject if Justice? In: Hans-Georg Soeffner (Hrsg.): Unsichere Zeiten. Wiesbaden, 717-733.

Global Commission on International Migration (2005): Bericht der Weltkommission für international Migration. Berlin.

Hess, Sabine/Tsianos, Vassilis (2004): Europäisierungder Migrations-und Grenzpolitiken. (http:// www.transitmigration.org/homearchiv.html).

Ignatieff, Michael (2002): Die Politik der Menschenrechte. Berlin.

Koenig, Mattias (2005): Weltgesellschaft, Menschenrechte und der Formwandel des Nationalstaates. In: Bettina Heinz/Richard Münch/Hartmann Tyrell (Hrsg.): Weltgesellschaft. Theoretische Zugänge und empirische Problemlagen. (Sonderheft der Zeitschrift für Soziologie). Stuttgart, 374-393.

Luhmann, Niklas (1997): Die Gesellschaft der Gesellschaft. Frankfurt.

Mau, Steffen/Burkhardt, Christoph (2010): Zuwanderung und die Ressourcen wohlfahrtsstaatlicher Solidarität. In: Hans-Georg Soeffner (Hrsg.): Unsichere Zeiten. Wiesbaden, 141-155.

Mead, George H. (1934/1968): Geist, Identität und Gesellschaft. Frankfurt/M.

Offe, Claus (2011): From Migration in Geographic Space to Migration in Biographic Time: Views from Europe. In: The Journal of Political Philosophy, Vol 19, No. 3, 333-373.

Paleologo, Fulvio Vassalo (2008): Einleitung. In: Gabriele del Grande: Mamadous Fabrt in den Tod. Karlsruhe, 9-17.

Pogge, Thomas (2011): Weltarmut und Menschenrechte. Stuttgart.

Ritsert, Jürgen (2009): Der Mythos der nicht-normativen Kritik. In: Ders.: Probleme der Dialektik heute. Wiesbaden, S. 161-176.

Sachar, Ayelet (2009): The Birthright Lottery: Citizenship and Global Inequality. Harvard University Press.

Scherr, Albert (2010): Diskriminierung und soziale Ungleichheiten. In: Hormel, Ulrike/Scherr, Albert (Hrsg.): Diskriminierung. Wiesbaden, S. 35-60.

- (2012): Nationalstaatlichkeit, Moral und Kritik. In: Sozialwissenschaftliche Literaturrundschau, H. 1/12, S. 27-36.

Schimank, Uwe (2005): Weltgesellschaften und Nationalgesellschaften. In: Bettina Heinz/Richard Münch,/Hartmann Tyrell (Hrsg.): Weltgesellschaft. Theoretische Zugänge und empirische Problemlagen. (Sonderheft der Zeitschrift für Soziologie). Stuttgart, S. 394-414.

Stichweh, Rudolf (2000): Die Weltgesellschaft. Frankfurt .

Walzer, Michael (1992): Sphären der Gerechtigkeit. Frankfurt/New York.

Weinzierl, Ruth/Lisson, Urszula (2007): Grenzschutz und Menschenrechte. Eine europarechtliche und seerechtliche Studie. Berlin: Deutsches Institut für Menschenrechte. 